\title{
Article \\ A Multi Objective Evolutionary Algorithm for the Parameters Extraction of Organic Thin Film Transistors Models
}

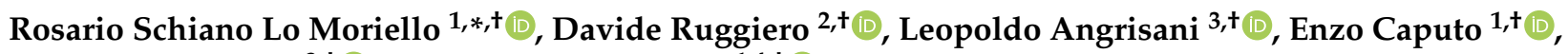 \\ Francesco de Pandi ${ }^{3,+}$ and Giorgio de Alteriis ${ }^{1,4,+}$ (iD \\ 1 Dipartimento di Ingegneria Industriale, Università degli Studi di Napoli Federico II, 80125 Naples, Italy; \\ enzo.caputo@unina.it (E.C.); giorgio.dealteriis@unibg.it (G.d.A.) \\ 2 AMS R\&D, STMicroelectronics, Arzano, 80022 Naples, Italy; davide.ruggiero@st.com \\ 3 Dipartimento di Ingegneria Elettrica e delle Tecnologie dell'Informazione, Università degli Studi di Napoli \\ Federico II, 80125 Naples, Italy; angrisan@unina.it (L.A.); francesco.depandi@unina.it (F.d.P.) \\ 4 Department of Management, Information and Production Engineering, University of Bergamo, Dalmine, \\ 24044 Bergamo, Italy \\ * Correspondence: rschiano@unina.it; Tel.: +39-081-768-3866 \\ + These authors contributed equally to this work.
}

check for

updates

Citation: Schiano Lo Moriello, R.; Ruggiero, D.; Angrisani, L.; Caputo, E.; de Pandi, F.; de Alteriis, G. A Multi Objective Evolutionary Algorithm for the Parameters Extraction of Organic Thin Film Transistors Models. Electronics 2021, 10, 939. https:// doi.org/10.3390/electronics10080939

Academic Editor: Yahya Moubarak Meziani

Received: 23 March 2021

Accepted: 12 April 2021

Published: 14 April 2021

Publisher's Note: MDPI stays neutral with regard to jurisdictional claims in published maps and institutional affiliations.

Copyright: (c) 2021 by the authors. Licensee MDPI, Basel, Switzerland. This article is an open access article distributed under the terms and conditions of the Creative Commons Attribution (CC BY) license (https:/ / creativecommons.org/licenses/by/ $4.0 /)$.

\begin{abstract}
Thanks to their peculiar features, organic transistors are proving to be a valuable alternative to traditional semiconducting devices in several application fields; however, before releasing their exploitation, simulating their behaviour through adequate circuital models could be advisable during the design stage of electronic circuits and/or boards. Consequently, accurately extracting the parameter value of those models is fundamental to developing useful libraries for hardware design environments. To face the considered problem, the authors present a method based on successive application of Single- and Multi-Objective Evolutionary Algorithm for the optimal tuning of model parameters of organic transistors on thin film (OTFT). In particular, parameters are first roughly estimated to assure the best fit with the experimental transfer characteristics; the estimates are successively refined through the multi-objective strategy by also matching the values of the experimental mobility. The performance of the method has been assessed by estimating the parameters value of both P-type and N-type OTFTs characterized by different values of channel lengths; the obtained results evidence that the proposed method can obtain suitable parameters values for all the considered channel lengths.
\end{abstract}

Keywords: organic transistors on thin film; model parameters estimation; multi-objective optimization; evolutionary algorithms

\section{Introduction}

In recent decades, organic field effect transistors (OFETs) have proved attractive to the scientific community due to the opportunity they offer in different areas of low-cost electronic application [1]. Several studies have been carried out to improve the performance of organic transistors, such as improving the semiconductor-insulating interface [2,3], the introduction of additional organic layers [4,5], the use of efficient contact materials [6,7] and the optimization of the manufacturing process $[8,9]$. The wide variety of organic semiconductors and their particular sensitivity to various elements and compounds make OTFTs a good candidate for low-cost selective chemical and physical sensors. Organic semiconductors, whose structure is mainly amorphous or at the most semicrystalline, are characterized by only a limited degree of molecular ordering [1]; due to the low mobility of the charge carriers, a limited performance can be achieved compared to those granted by single crystal devices.

Since high-temperature processes rapidly degrade organic compounds, manufacturing techniques have been developed to realize printed electronics. As a further advantage, 
the use of low temperatures reduces problems related to mechanical stress associated with different coefficients of thermal expansion of the involved materials. Moreover, organic molecules can be chemically modified to meet specifications that allow their use for:

- Power supply, as photovoltaic panels, batteries, fuel cells [10];

- Calculation and memory, in applications where silicon chips are too expensive, delicate or cumbersome [11];

- Flexible displays of electronic devices [12];

- Other components, from Radio Frequency Identification (RF-ID) sensors to laser printers, up to light detectors [13].

To preliminarily verify the improvement and the behaviour of the organic devices in complex electronic circuits and/or boards, a preliminary simulation stage involving proper environments for computer assisted design is advisable. In this environment, each component of the circuit is simulated according to a circuital model capable of replicating its operation; the better the compliance of the model to real behavior, the better the predictions of how the devices work [14]. This way, the development of circuit models capable of simulating the behavior of organic transistors within the hardware design environment is needed; in other words, accurately estimating the value of the physical/geometric parameters that define the mathematical and circuit model of such devices is mandatory.

This estimation usually results in the formulation of an optimization problem for the parameter tuning of the circuital model; to address this problem, different approaches can be pursued. In particular, traditional optimization strategies, included in the so-called hard computing techniques, are currently outperformed by metaheuristic approaches, usually referred to as soft computing [15]. As discussed in [16] soft computing techniques can easily overcome typical drawbacks affecting hard computing solutions (as an example, local maximum trapping or uncertain mathematical model) and prove particularly suitable for multi-dimensional nonlinear optimization problems.

Among the soft computing techniques, the evolutionary algorithm proves to be suitable to force and solve the considered problems. In particular, evolutionary algorithms represent a class of stochastic search algorithms for optimization problems that rely on the imitation of those biological processes that allow populations of organisms to adapt to the environment in which they live.

In order to tuning the parameters of a defined model, two different approaches can be followed:

- Define the problem as a single-objective problem, where parameters values are obtained $\mathrm{b}$ by minimizing/maximizing a scalar function (usually referred to a goal function) dependent on one or more variables;

- Define the problem as a multi-objective problems, where the optimal values of the parameters are evaluated by finding the minimum/maximum of multiple objective functions, usually conflicting with one another.

With specific regard to parameter extraction for OTFT models, several valuable techniques, such as the Queen-Bee (genetic type algorithm) [17] or multi objective evolutionary algorithms (MOEA) techniques [18-21], have recently been proposed.

To further improve the estimate of the circuit model parameters, the authors propose a new method based on the successive application of single-objective and multi-objective problems exploiting the Differential Evolution (DE) as optimization algorithm [22]. More specifically, a first application of single objective optimization is mandated to carry out a rough but fast estimation of the model parameters by fitting the experimental curves of OTFT transfer characteristics. Obtained estimates are exploited as a first guess of the successive multi-objective optimization problem; in particular, parameter values are determined in such a way as to simultaneously match the experimental mobility of the device.

For the adopted evolutionary algorithms, the authors focus their attention on DE due to the offered improvements in terms of stability and convergence with respect to other available solutions (for example, Particle Swarm Optimization (PSO)). In particular, DE 
shows a more stable behavior (PSO is more sensitive to parameter changes than DE), fewer iterations and better solutions of the problems $[23,24]$.

The paper is organized as follows: Sections 2 and 3 summarizes the fundamental concepts of OTFT and MOEAs theory, respectively; Section 4 presents the adopted OTFT model; Section 5 describes the proposal; Section 6 illustrates the results obtained applying the proposed approach on actual organic transistors; Section 7, finally, gives concluding remarks.

\section{OTFT Structure and Operating Principle}

The most common structure for OFETs is the Organic Thin Film Transistors (OTFT), a three-terminal device, in which the gate voltage controls the flow of current between source and drain, as in traditional metal-oxide-semiconductor field-effect transistors (MOSFETs).

As shown in the simplified structure of Figure 1, a thin layer of organic semiconductor is deposited on top of one of insulator.

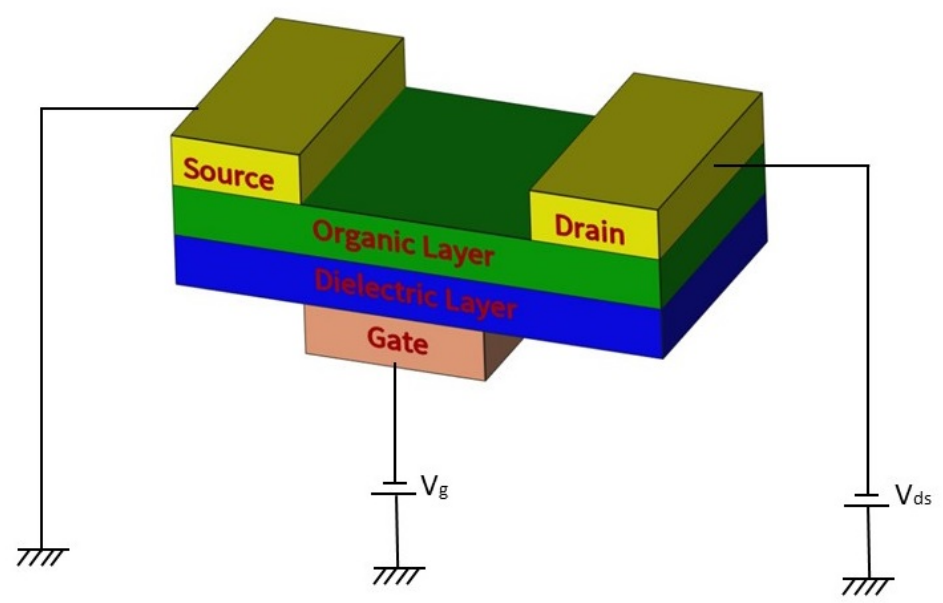

Figure 1. The architecture of organic TFT are similar, in terms of structures and functionalities, to that of traditional MOSFET.

According to the so-called Bottom-Gate-Top-Contact structure, gate electrode is below the insulator, while source and drain contacts are placed above the semiconductor. Figure 2 shows other possible configurations depending on the relative positions of gate, source and drain.

The organic material most used to make the channel of OTFTs is pentacene. It is characterized by an electron mobility much lower than that of holes; therefore, pentacene is used as a p-type material. Its electronic affinity is within the range from 2.8 to $3.2 \mathrm{eV}$, while the forbidden energy gap extends between 1.8 and $2.2 \mathrm{eV}[25,26]$. The conduction in an organic transistor occurs in a very thin region (a few nanometers, corresponding to a couple of molecular layers) at the insulator-semiconductor interface. As a consequence, granting a uniform and regular deposition of pentacene turns out to be necessary, at least in this region.

As stated above, the semiconducting behavior of an organic transistor is obtained through a thin layer of organic molecules or conjugated polymers, which is almost intrinsic. In particular, N-channel OTFTs have n-type substrate and turn on as soon as electrons are accumulated under the dielectric; on the contrary, P-channel OTFTs have the p-type substrate and light up, accumulating a layer of holes (majority charge carriers) under the dielectric.

A surface of organic material does not have a sufficiently high concentration of free carriers; therefore, in the absence of a gate voltage that induces a layer of free charge at the interface, the organic material behaves almost like an insulator. 

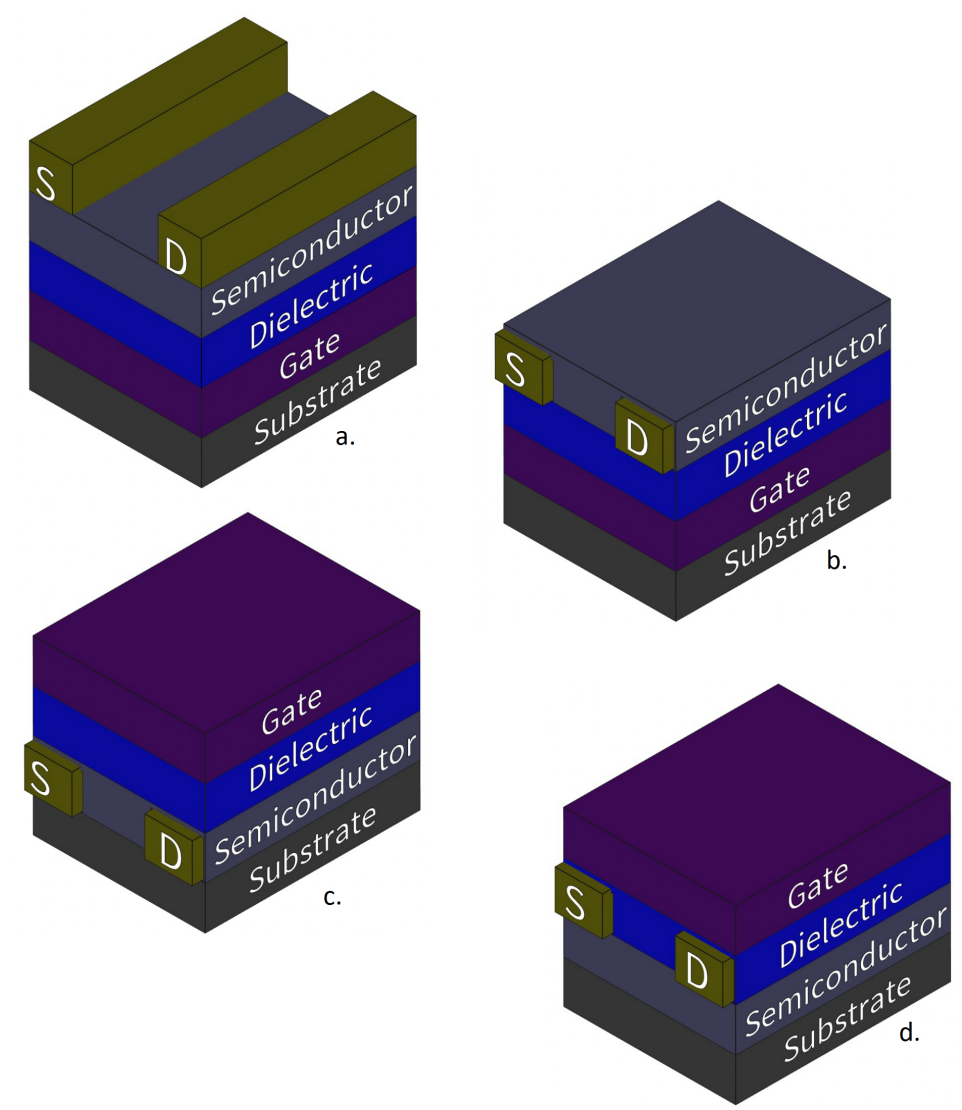

Figure 2. Different architectures of OFETs. (a) top-contact bottom-gate, (b) bottom-contact bottomgate, (c) bottom-contact top-gate and (d) top-contact top-gate.

OTFTs differ significantly from inorganic semiconductor MOSFETs in terms of operating regime [27]. In the on state, MOSFETs, in fact, operate in inversion mode because the charge carriers in the conducting channel have opposite signs with respect to the majority charge carriers in the bulk. The conductive channel is formed only after the gate voltage has exceeded a limit value, which is defined as the threshold voltage VT of the transistor.

On the contrary, OTFTs operate only in the accumulation regime; when a positive voltage is applied to the gate, a quantity of negative charge is, in fact, induced at the source, in such a way as to form an energy barrier for the electrons [28]. The low currents visible in the off state are thus only due to losses through the gate dielectric. Accumulation involves significant differences between OTFTs and MOSFETs. The source and drain of the OFETs must be made using materials whose work functions allow the charge transport.

Differently from MOSFETs, the substrate is not conductive in organic TFTs, since the semiconductor has a very low concentration of carriers. The organic film only transports what is injected; for this reason, in an OTFT, rectifying contacts are not necessary between source (drain) and substrate. On the contrary, ohmic contacts, i.e., contacts characterized by low resistance, are involved. Good-quality ohmic contacts constitute a technologically very important issue that can severely affect the characteristics of the devices.

Ohmic contacts on pentacene (or even rectifying contacts exhibiting low barrier height) are realized by means of metals characterized by high values of work function $W_{f}$ (as an example gold with $5.10 \mathrm{eV}$ ).

Regarding the performance of an organic TFT, the most influencing parameters are:

- The mobility of the channel carriers, which is closely related to the switching speed of the transistor;

- The $I_{O N} / I_{\text {OFF }}$ ratio, i.e., the ratio between the transistor on and off currents; it is usually exploited as figure of merit for having a high performance (more $I_{O N}$ ) and low leakage power (less $I_{O F F}$ ) for transistors; 
- The slope in subthreshold region, which indicates the voltage excursion necessary to reduce the current by a factor of 10 .

\section{Soft Computing Algorithms}

Differently from traditional processing methods (typically referred to as hard computing), soft computing aims at adapting to the inaccuracy or uncertainty usually associated with modeling the real world. To this aim, soft computing approaches take advantage from their main pillars:

$\diamond \quad$ the capability of modeling and controlling uncertain and complex systems;

$\diamond \quad$ the reduced need for explicit knowledge in a particular domain, since soft computing methods do not call for wide-ranging mathematical formulation pertaining to the problem;

$\diamond \quad$ in optimization problems, the solutions can be prevented from being stuck into local minima by using global optimization strategies;

$\diamond \quad$ the capability of learning and approximating complex functional relationships of neural networks.

If hard and soft computing strategies for processing and calculation are compared, the differences given in Table 1 can easily be outlined.

Table 1. Comparisons between hard and soft computing strategies.

\begin{tabular}{ll}
\hline Hard Computing & Soft Ccmputing \\
\hline Rigidly programmed & Trainable and adaptive operations \\
\hline Based on binary logic & Based on fuzzy logic \\
\hline Deterministic & Stochastic \\
\hline $\begin{array}{l}\text { Based on precisely stated analytical } \\
\text { model }\end{array}$ & $\begin{array}{l}\text { Tolerant to imprecision, uncertainty, and ap- } \\
\text { proximation }\end{array}$ \\
\hline Series operations & Parallel operations \\
\hline Provide precise results & Provide approximated results \\
\hline
\end{tabular}

Among the available solutions based on soft computing, the authors focused their attention on evolutionary algorithms, which are stochastic strategies mainly used for the optimization of parametric mathematical models.

The operating steps of evolutionary algorithms are based on the concept of population. Each point of the domain of the function to be optimized can be considered as an "individual" who has a greater or lesser ability to adapt to the surrounding environment, as in the principle of Darwinian natural selection. The degree of adaptation of each individual is measured by means of a function usually referred to as fitness function.

The algorithm operates in such a way as to allow the population of individuals to be able to increase in number or change their characteristics so that the algorithm can explore new spaces in the research domain. This apparently simple mechanism has proved to be of extraordinary power; evolutionary algorithms are, in fact, used to solve a large number of problems in engineering and scientific fields.

The evolutionary algorithms, unlike the standard search procedures, have the advantage of not requiring that the fitness functions are differentiable. Furthermore, they are global search techniques for an optimal and non-local solution and, therefore, do not depend on the initial conditions (unlike some classic methods such as the simplex algorithm).

\subsection{Mono and Multi-Objective Evolutionary Algorithms}

In the application field of optimization, a distinction is made between:

- Mono-objective problems, aiming at evaluating the optimal value of a scalar function (referred to as objective function) which is dependent on one or more variables. 
- Multi-objective problems, in which the best of several objective functions, generally in contrast to one another, is pursued.

Evolutionary algorithms are extremely effective in solving multi-objective problems because they can simultaneously take a large set of solutions (individuals of the population) into account.

In the paper, a particular MOEA, referred to as Multi-Objective_Differential Evolution (MODE), was used. The MODE approach is tailored on a two-goal optimization problem thanks to the exploited Differential Evolution algorithm.

Differential Evolution allows to provide an efficient solution to the following problem: given a fitness function $F(y)$, defined on a multi-dimensional space of vectors $y$, determine the vector $\hat{y}$ in which $F$ assumes its optimal (either minimum or maximum) value, $F(\hat{y})$. The DE is a stochastic algorithm whose difference from other evolutionary algorithms lies in the way in which the search for the optimum is carried out in the space of possible solutions. In fact, the evolution of the DE is not based on the output of a default probability distribution function, but is driven by differences between candidate solutions.

The DE operates on a population of individuals, each of which can be schematized as a point in the search space, characterized by two parameters: a vector $y$ of the parameters, and the corresponding fitness value $F(y)$ (which represents the measure of the goodness of the solution to the problem)

$$
a=(y, F(y))
$$

The main operating steps of the DE algorithm are schematized both in the flowchart reported in Figure 3 and described below.

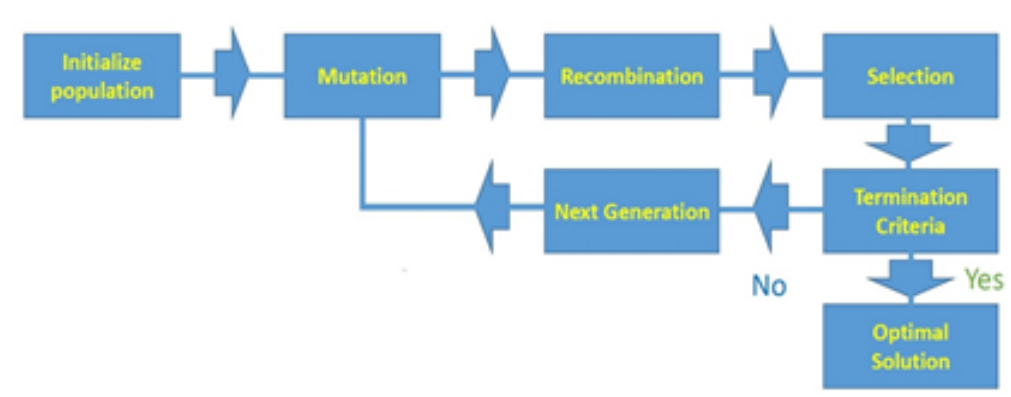

Figure 3. Block diagram of the operating steps of the differential evolution algorithm.

\subsection{Differential Evolution Algorithm}

As stated above, DE is a population-based algorithm; let us consider a population of $\mathrm{N}$ individuals drawn from the whole search space

$$
x_{(\mathrm{n}, \mathrm{i})}{ }^{g}=\left[x_{(\mathrm{n}, 1)^{g}}, x_{(\mathrm{n}, 2)^{g}}, x_{(\mathrm{n}, 3)^{g}}, \ldots, x_{(\mathrm{n}, \mathrm{D})^{g}}\right]
$$

where $g$ is the current individual generation, $\mathrm{i}=1,2, \ldots, \mathrm{D}$ stands for the index identifying the parameters, $\mathrm{D}$ is the cardinality of the vector of parameters to be optimized and $\mathrm{n}=1$, $2, \ldots, N$.

\subsubsection{Initial Population}

Initial population is randomly generated in such a way as each parameter of the individual belongs to the interval defined by its corresponding lower and upper bounds

$$
x_{(\mathrm{n}, \mathrm{i})}=x_{\mathrm{i}}^{L}+\operatorname{rand} *\left(x_{\mathrm{i}}^{U}-x_{\mathrm{i}}^{L}\right)
$$

where $x_{\mathrm{i}}^{L}$ and $x_{\mathrm{i}}{ }^{U}$ are respectively the lower bound and upper bound of the $i$-th parameter of the vector $x_{\mathrm{n}}$. 


\subsubsection{Mutation}

For each individual of the current population, three vectors $x_{\mathrm{rjn}}{ }^{g}(j=1,2,3)$ are randomly selected among the current generation, and a further vector is obtained from their weighted combination as

$$
v_{\mathrm{n}}^{g+1}=x_{\mathrm{r} 1 \mathrm{n}}^{g}+W\left(x_{\mathrm{r} 2 \mathrm{n}^{g}}^{g}-x_{\mathrm{r} 3 \mathrm{n}^{g}}\right) \quad n=1,2,3 \ldots N
$$

$v_{n}{ }^{g+1}$ is the so-called donor vector and $W \in[0,1]$.

\subsubsection{Recombination}

A fundamental step of each evolutionary algorithm is the recombination phase, responsible for their ability to efficiently investigate the whole solutions space. As for the adopted DE approach, a trial vector $\left.u_{(\mathrm{n}, \mathrm{i})}\right)^{g+1}$ is derived from both the target vector $x_{(\mathrm{n}, \mathrm{i})^{g}}$ and the donor vector $v_{(\mathrm{n}, \mathrm{i})}{ }^{g+1}$ according to

$$
\left.u_{(\mathrm{n}, \mathrm{i})}\right)^{g+1}= \begin{cases}\left.v_{(\mathrm{n}, \mathrm{i})}\right)^{g+1} & \text { if rand } \leq C_{\mathrm{p}} \text { or } i=I_{\mathrm{rand}} \\ x_{(\mathrm{n}, \mathrm{i})^{g}} & \text { if rand } \geq C_{\mathrm{p}} \text { or } i \neq I_{\text {rand }}\end{cases}
$$

where $I_{\text {rand }}$ is a random integer number drawn within the interval $[1, D]$ and $C_{p}$ is a defined recombination probability. In other words, there is a defined probability that a specific parameter of the individual vector is changed and copied from the corresponding value of the donor vector.

\subsubsection{Selection}

The target vector $x_{(\mathrm{n}, \mathrm{i})}{ }^{g}$ is compared with the trial vector $\left.u_{(\mathrm{n}, \mathrm{i})}\right)^{g+1}$ and the one characterized by the lowest value of the fitness function is selected for the next generation

$$
x_{\mathrm{n}}{ }^{g+1}= \begin{cases}\left.u_{(\mathrm{n}, \mathrm{i})}\right)^{g+1} & \text { if } F\left(u_{\mathrm{n}}{ }^{g+1}\right)<F\left(x_{\mathrm{n}}{ }^{\mathrm{g}}\right) \\ x_{\mathrm{n}}{ }^{g} & \text { otherwise }\end{cases}
$$

\subsubsection{Termination Criteria}

The search algorithm executes the previously considered steps until a proper termination criterium is met. For the considered application, different criteria have been taken into account:

- Maximum number of iterations;

- Objective limit, i.e., minimum value of the objective function is reached;

- Maximum number of evaluation of the objective function;

- Function tolerance: The iteration ends when the relative changes in the objective function are below a threshold established by a non-negative value of this hyperparameter. This stopping condition is evaluated in a certain number of iterations established by a parameter, usually referred to as "patience" or "max stall iterations".

\section{Adopted OTFT Model}

The proposed approach is particularly tailored for P- and N-channel OTFTs realized by means of organic semiconductor and designed according to an interdigitated geometry. Considered devices are bottom contact top gate, whose layout is represented in Figures 4 and 5. To extract parameters that match with the transfer curves of an organic TFT, a compact parametric circuit model is chosen; the model has to satisfy the following specific requests:

- Must consistently represent the behavior of OTFTs;

- Must be analytical, without differentials or integrals expressions;

- Must be simple and easily derivable;

- Must have parameters that can be easily characterized;

- Must have relationships that are physically justifiable. 
Consequently, the equation of the model for the OTFT drain current must take the different operating regimes, i.e., (i) off-state, (ii) linear and (iii) saturation regions, into account. For these devices, the presence of an energy barrier at the metal-semiconductor region was hypothesized. This contribution can be considered as a Schottky diode placed on the TFT source contact. For short-channel devices $(\mathrm{L} \leq 100 \mu \mathrm{m})$, the presence of the Schottky diode is fundamental for accurately modeling electrical characteristics. In the absence of the diode, for low channel length, the current values obtained from the model, in fact, overestimate the experimental ones, due to the contact effects that influence the devices' performance.

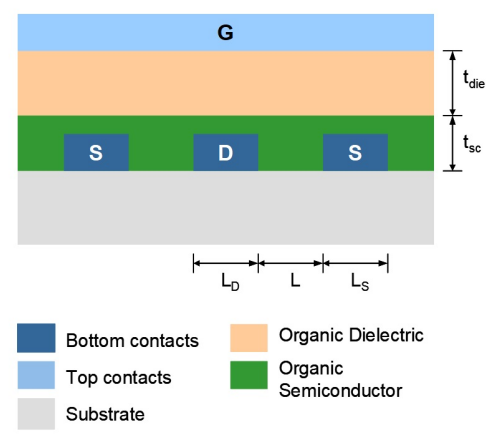

(a)

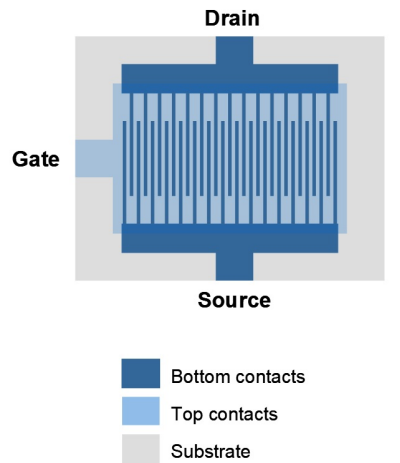

(b)

Figure 4. P-type OTFT layout. (a) side view and (b) lateral view.

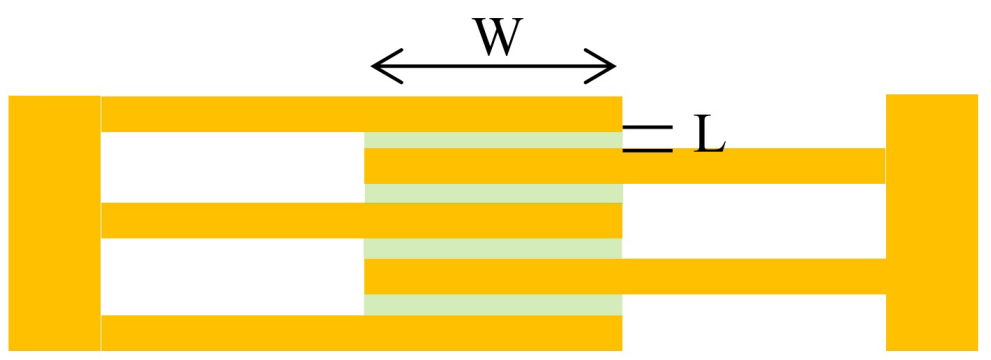

Figure 5. P-type OTFT multi-fingers structure of source-drain electrodes.

The corresponding models, associated with p-type and n-type, are shown in Figure 6a and Figure $6 \mathrm{~b}$, respectively. The compact circuit model consists of five nodes, indicated through the yellow arrows in the diagram. Three nodes ( $g$ - gate, $d$-drain, and $s$-source) are accessible from the outside, while two nodes (is and $i d$ ) are internal nodes. According to the sign of the potential drop from the drain to the source, the diode D0 is positioned in the branch-connecting node $s$ and node is or in the branch-connecting node $i d$ and node $d$.

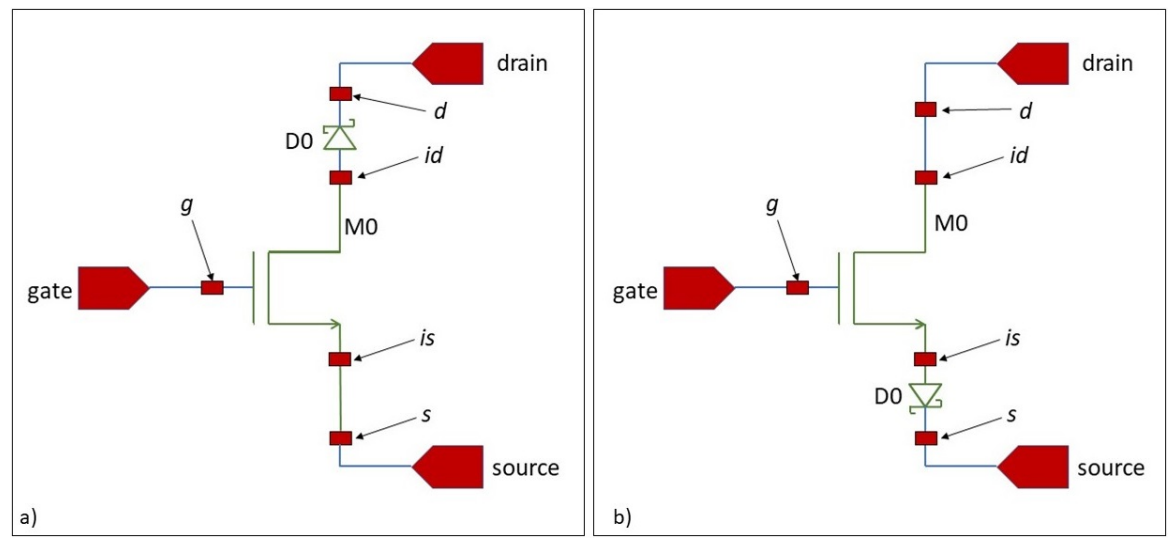

Figure 6. Circuit model for p-type (a) and n-type (b) OTFT, respectively. 


\subsection{OTFT IV Characteristic}

Let the voltage of the node $x$ be indicated as $V(x)$; in order to describe the electrical model of the OTFT, the following voltage drops have to be taken into account:

- $V_{\mathrm{gs}}=V(g)-V(s)$;

- $\quad V_{\mathrm{gd}}=V(g)-V(d)$;

- $\quad V_{\mathrm{ds}}=V(d)-V(s)$;

- $V_{\mathrm{si}}=V(i s)-V(s)$;

- $V_{\mathrm{di}}=V(i d)-V(d)$;

- $V_{\mathrm{dsTFT}}=V_{\mathrm{ds}}+V_{\mathrm{di}}-V_{\mathrm{si}}=V(g)-V(s)$.

In these assumptions, the transistor model can be expressed as

$$
\begin{gathered}
L_{\text {Early }}=L-d L V_{\mathrm{ds}} *\left|V_{\mathrm{dsTFT}}\right| \\
I_{\mathrm{Off}}=I_{00} * W_{\text {eff }} / L_{\text {Early }} * V_{\mathrm{dsTFT}} \\
I_{\mathrm{TFT}}=K_{1} * W_{\text {eff }} / L_{\text {Early }} *\left(V_{\mathrm{SS}} * \log \left(1+e^{\left.\left(V_{\mathrm{gs}}-V_{\mathrm{Si}}-V_{\mathrm{fb}}\right) / V_{\mathrm{SS}}\right)}\right)^{m}-\right. \\
\left(V_{\mathrm{SS}} * \log \left(1+e^{\left.\left(V_{\mathrm{gd}}-V_{\mathrm{di}}-V_{\mathrm{fb}}\right) / V_{\mathrm{SS}}\right)}\right)^{m}+I_{O} f f\right.
\end{gathered}
$$

The 6 parameters to be estimated are:

- $\quad K_{1}$ and $m$, which depend on the charge mobility;

- $\quad V_{\mathrm{fb}}$, which depends on the gate voltage;

- $\quad V_{\mathrm{SS}}$, which depends on the subthreshold slope;

- $I_{00}$, which depends on the off current of the device;

- $d L V_{\mathrm{ds}}$, which is adopted to suitably scale the channel length according to the drain and source voltage.

The parameter $L_{\text {Early }}$ has been considered to take into account the channel length $L$ variation associated with the voltage $V_{\mathrm{ds}}$, while $W_{\text {eff }}$ represents the effective channel width. Regarding the diode, the following model has been adopted

$$
\begin{gathered}
I_{\text {rev }}=I_{00 \mathrm{~d}} * W_{\text {nom }} *\left(\left(V_{\mathrm{gs}} / V_{00}\right)^{\beta}+\left(V_{\mathrm{gmin}} / V_{00}\right)^{\beta}\right) * e^{\left(V_{\mathrm{si}} / V_{\text {diode }}\right)^{\alpha}} \\
I_{\text {diode }}=-I_{\text {rev }} *\left(e^{-q * V_{\mathrm{si}} / \eta k T}\right)
\end{gathered}
$$

The corresponding fit parameters of the Schottky diode are $\alpha, \beta, \eta, V_{\text {diode }}, I_{00 \mathrm{~d}}, V_{\mathrm{gmin}}$, which are usually referred to as contact parameters. $V_{\text {diode }}$ is just a constant contact parameter and not the voltage across the diode, the factor $e^{\left(V_{\mathrm{si}} / V_{\text {diode }}\right)^{\alpha}}$ takes the Schottky barrier reduction induced by the electric field at the source contact into account. The factor $\left(V_{\mathrm{gmin}} / V_{00}\right)^{\beta}$ is an empirical expression to fit the $V_{\mathrm{gs}}$ dependence of diode reverse current [29].

\subsection{OTFT Mobility Model}

To further improve the estimates of the previous parameters, the charge mobility has been modeled in both linear and saturation operating regimes. In particular, the following expressions hold

$$
\begin{aligned}
& \mu_{\mathrm{SAT}}=\left(\frac{2 L_{\mathrm{Early}}}{W C_{\mathrm{OX}}}\left(\frac{d \sqrt{I_{\mathrm{d}}}}{d V_{\mathrm{gs}}}\right)\right)_{\text {max }}^{2} \\
& \mu_{\mathrm{LIN}}=\frac{L_{\text {Early }}}{W C_{\mathrm{OX}} V_{\mathrm{ds}}}\left(\frac{d I_{\mathrm{d}}}{d V_{\mathrm{gs}}}\right)_{\text {max }}
\end{aligned}
$$


where $W$ is the channel width, $C_{O X}$ is the capacity of the gate oxide. Equations (12) and (13) have been deduced from the traditional formulas of transistors in the assumptions that the following OTFT models hold

$$
\begin{aligned}
& I_{\mathrm{d}}=\frac{1}{2} \frac{W}{L} \mu C_{\mathrm{OX}}\left(V_{\mathrm{gs}}-V_{\mathrm{T}}\right)^{2} \\
& I_{\mathrm{d}}=\frac{W}{L} \mu C_{\mathrm{OX}}\left(V_{\mathrm{gs}}-V_{\mathrm{T}}\right) V_{\mathrm{ds}}
\end{aligned}
$$

in the saturation and linear regime, respectively.

It is worth noting that models for IV characteristics and mobility are tightly coupled, since the parameters needed to model the mobility are first derived from the IV characteristics model.

\section{Proposed Method}

The method aims at estimating the whole set of model parameters capable of assuring the best concurrence between experimental and simulated evolution of both transfer and output characteristics, as well as between experimental and simulated mobility for all the available channel lengths at different values of voltage polarizations.

The most suitable solution consisted of two successive steps, the first of which exploits a mono-objective problem to gain a rough estimate of the parameters of the OTFT model for the IV characteristics. A successive MOEA approach exploiting DE algorithms allows a simultaneous estimation of the carriers' mobility to be carried out, thus making it possible to obtain model parameters suitable for all the available channel lengths. In this respect, each element of the DE population consists of a possible solution, whose entries (usually referred to as genes) are the model parameters to be estimated.

As for the first estimation procedure, the iterative process enlisted the following steps:

1. Search for the parameter set of the transistor and the diode;

2. Estimation, for each $V_{g}$ and $V_{d}$, of the corresponding voltage value $V_{s}$ of the diode;

3. Verification of the relationship $I_{\mathrm{tft}}=I_{\text {diode, }}$ since the same current in transistors and diodes flows, due to their series connection;

4. Verification of the found solution through the estimate of the measurement error acting as a fitness function between the experimental data and those simulated by the model. In particular, the root-mean-square error of the point-to-point difference between experimental and estimated characteristics have been adopted.

Once the OTFT mono-objective problem has been roughly determined, the authors applied a multi-objective optimization approach, considering mobility as an additional reference parameter. As well as searching the optimal parameter estimates that improve the fitting between the experimental and simulated I-V curves, the method evaluates the best values of parameters of the model (Equations (12)-(15)) in such a way as to minimize the difference between experimental and estimated mobility (the second fitness function). To this aim, the Multi Objective Differential Evolution (MODE) algorithm was used, which exploits, in a framework of multi-objective optimization, the parallel execution of several DE algorithms that were described before.

MODE is a multi-population, multi-objective DE approach. The algorithm can be summarized as follows: An initial population is generated at random. All dominated solutions are removed from the population using the Non-Dominated Sorting approach [16].

The remaining non-dominated solutions (i.e., those solutions located at the so-called Pareto front) are retained for recombination. Three parents are selected at random. A child is generated from the three parents and is placed into the population if it dominates the first selected parent; otherwise, a new selection process takes place. The schematic representation of MODE algorithm using DE approach is presented in Figure 7. 


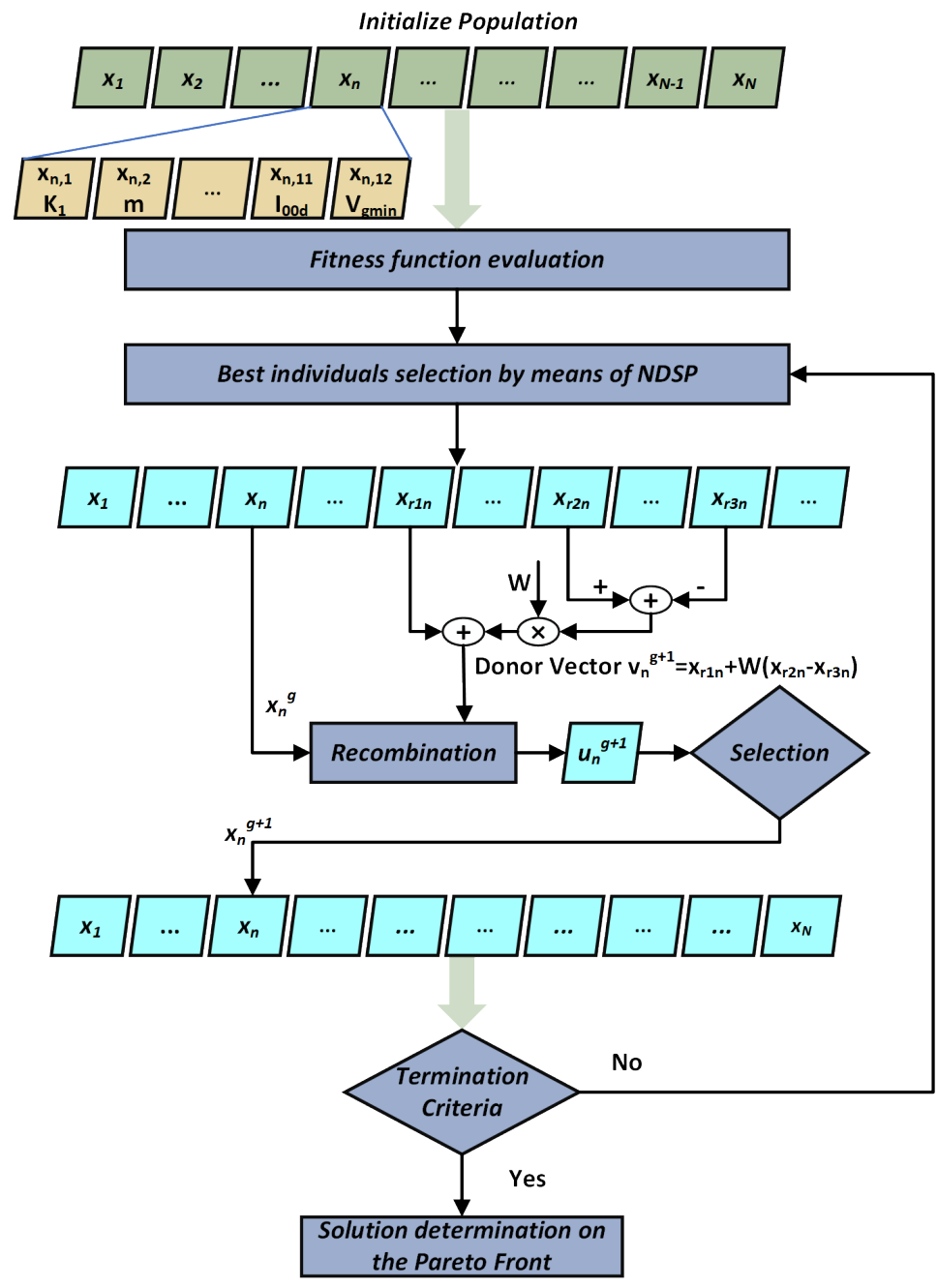

Figure 7. Block diagram of MODE algorithm with DE approach; selection phase is carried out by considering both fitness functions.

6. Method Validation

\subsection{Experimental Setup}

The experimental data of the OTFTs IV characteristics to be approximated by means of the considered models were obtained through a suitable measurement setup. In particular, the measurement station enlisted

- Probe-station PM5 Karl Suss MicroTec;

- Semiconductor device analyzer B1500A by Agilent Technologies;

- LCR Meter 4184 by Agilent Technologies.

The probe station (Figure 8) consists of a circular base, suitably perforated and shaped, on which the device under examination is arranged. 


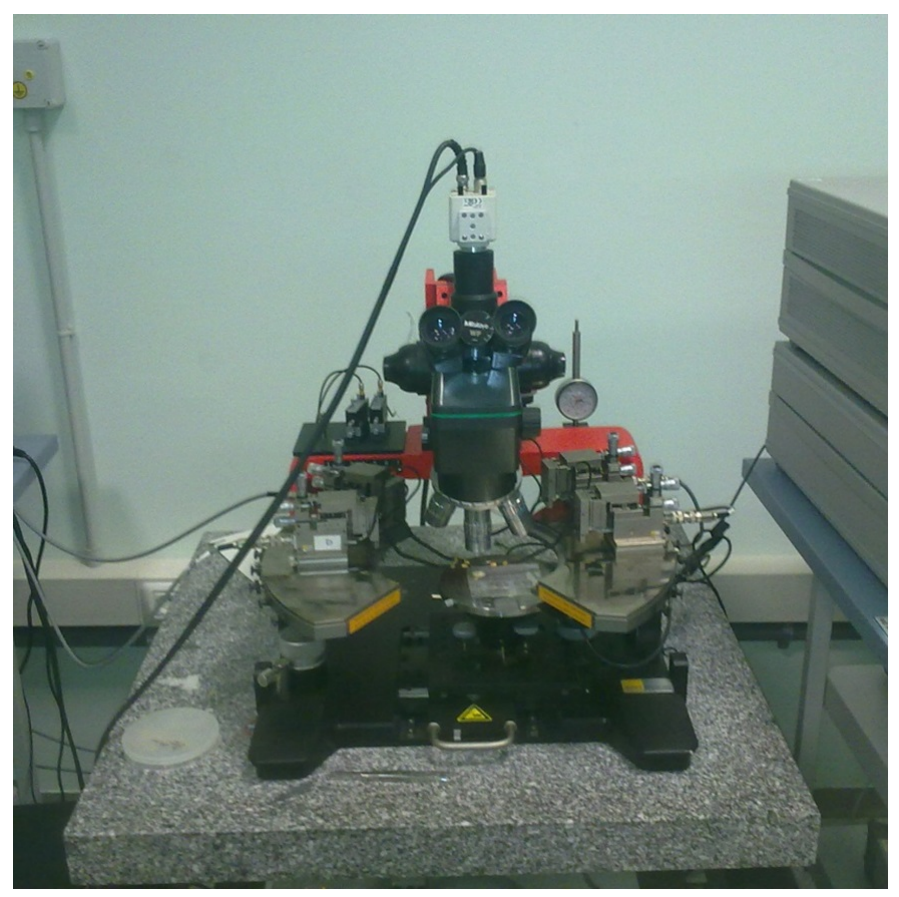

Figure 8. PM5 Karl Suss MicroTec.

The probe is connected to a vacuum system that, thanks to a system of holes in the circular base, ensures the maximum stability of the device under test; this is a key issue to be taken into account to assure sub-micrometric displacement of the device during the tests. The adhesion between the transistor under test and the circular base is obtained by a means of membrane pump that allows the limit pressure of $100 \mathrm{~Pa}$ to be reached. Moreover, the probe station in equipped with a micromanipulator system to position three needles on the source, drain and gate contacts. To this aim, a microscope is installed on the supports of the mobile station, and a camera is connected to its eyepiece (Figure 9).

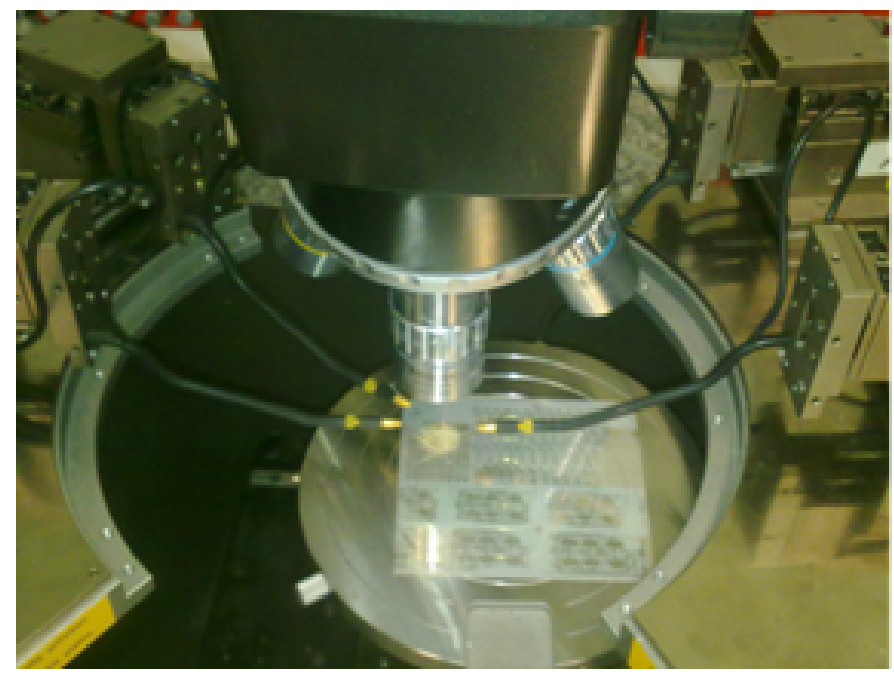

Figure 9. Probe with device under test already mounted.

The Agilent Technologies Semiconductor Device Analyzer B1500A (Figure 10) is used for the determination of static transfer and output characteristics. 


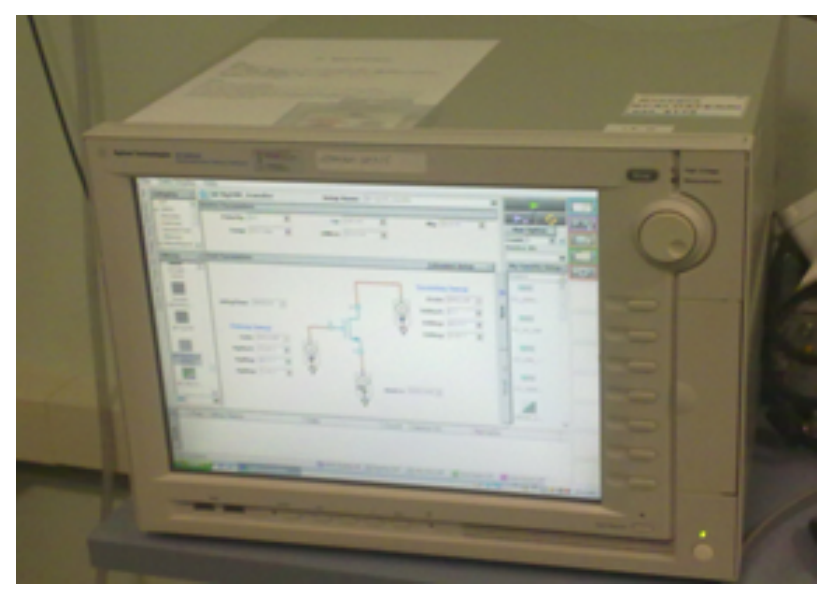

Figure 10. Semiconductor device analyzer.

The SDA is a precision instrument for determining the parameters of semiconductor devices, allowing the measurement of voltages and currents, the analysis, processing and graphic display of the characteristics of a large variety of multi-terminal electrical devices (e.g., diodes, bipolar transients, FETs, MOSs).

This instrument interfaced with the abovementioned LCR meter can also measure the capacitive contributions.

\subsection{Results}

The performance of the proposed approach has been assessed in a number of tests involving different devices. Tests have been conducted on P-channel and N-channel OTFT realized by the French research institute CEA-Liten. In the following, some results are reported to highlight the experienced efficacy.

\subsubsection{Performance Comparison between Mono- and Multi-Objective Algorithms}

As an example, the performance enhancement brought by the MODE approach is given in Figures 11 and 12, where the estimates of the transfer curve of an N-channel OTFT characterized by a channel length of $100 \mu \mathrm{m}$ are shown. As stated above, a first, fast but rough estimation of the parameters of the OTFT are gained by means of a mono-objective approach. The obtained estimates are exploited as first guess for the application of the multi-objective stage; as can be seen in Figure 12, the best improvement is related to the curves corresponding to a drain voltage equal to $70 \mathrm{~V}$, i.e., the one characterized by the worst result in the mono-objective approach. Similar behaviors have been experienced in tests conducted on the other values of channel length.

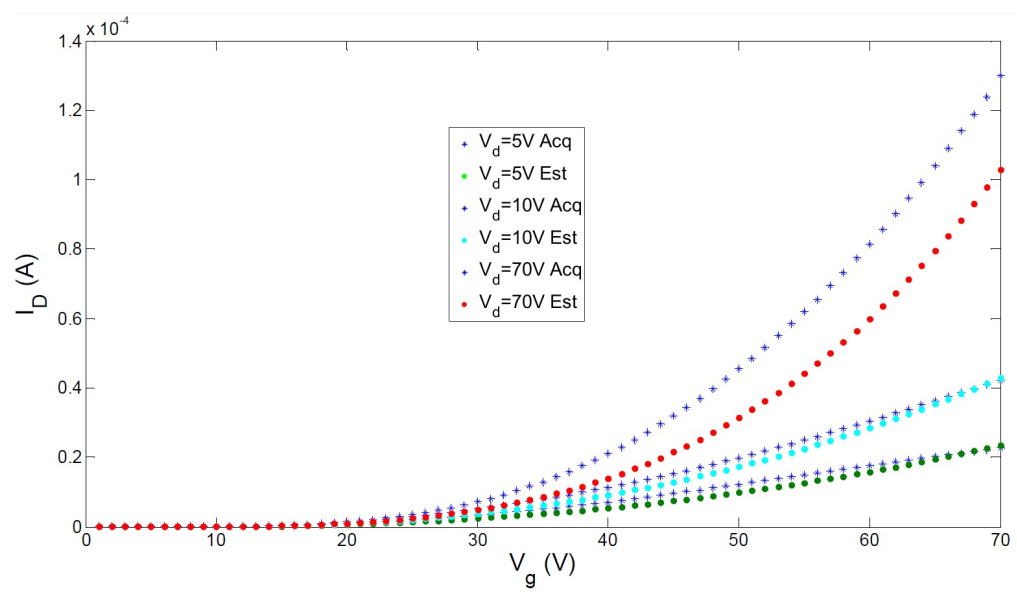

Figure 11. Comparison between acquired transfer curves and those estimated by means of monoobjective evolutionary approach. 


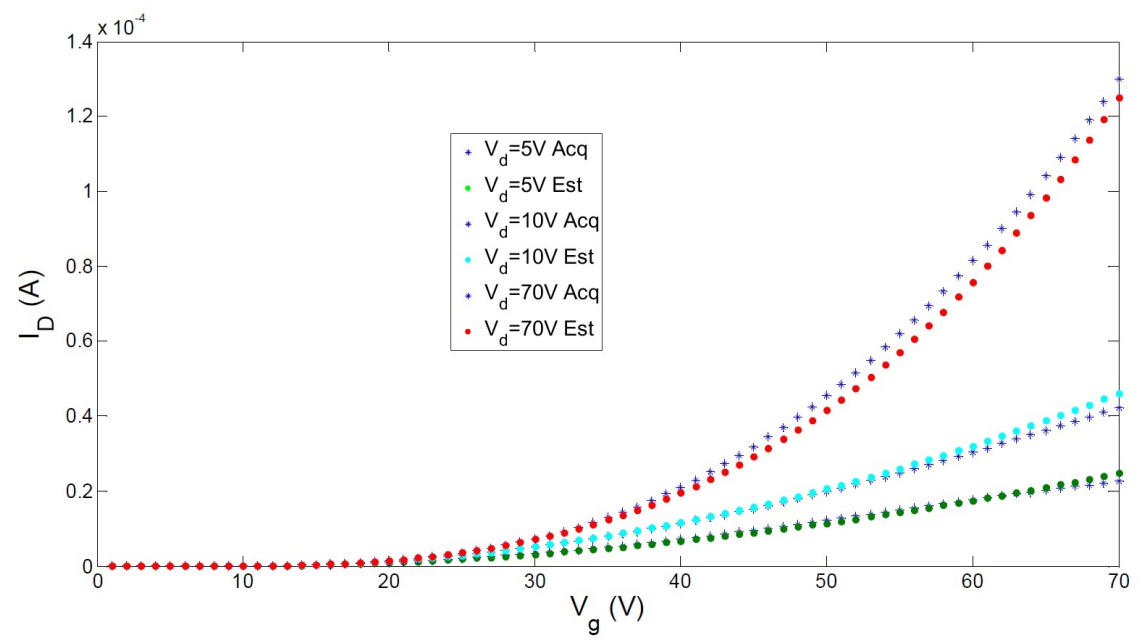

Figure 12. Comparison between acquired transfer curves and those estimated by means of multiobjective evolutionary approach.

\subsubsection{Mobility Estimation}

The mobility values, both in linear and saturation regime, are estimated according to Equations (12) and (13); the required current values were determined by means of the solution of mono-objective problem. In a similar way, the experimental mobility values were obtained through the same equations; as regards the currents, the values achieved during the experimental tests have been exploited.

For each acquired signal of each analyzed OTFT device, the greatest value of mobility, associated either with saturation of linear regime, is considered as representative for the device; this is the same approach as was followed for the values obtained from the experimental curves. For the sake of the brevity, mobility values estimated by means of the proposed method and those evaluated by the experimental data are compared in Tables 2 and 3 for only the CEA1298 N- and P-type OTFT device; a notable concurrence can be seen.

Table 2. Comparison between experimental and estimated data for CEA1298 N-type.

\begin{tabular}{llll}
\hline $\mathbf{L}[\boldsymbol{\mu m}]$ & V_DS & EXP & EST \\
\hline $\mathbf{1 0 0}$ & 5 & 1.04 & 1.09 \\
& 10 & 1.14 & 1.18 \\
& 70 & 0.72 & 0.76 \\
\hline $\mathbf{5 0}$ & 5 & 1.08 & 1.01 \\
& 10 & 1.01 & 0.90 \\
& 70 & 0.50 & 0.49 \\
\hline $\mathbf{2 0}$ & 5 & 0.71 & 0.60 \\
& 10 & 0.65 & 0.59 \\
& 70 & 0.20 & 0.22 \\
\hline
\end{tabular}


Table 3. Comparison between experimental and estimated data for CEA1298 P-type.

\begin{tabular}{llll}
\hline $\mathbf{L}[\boldsymbol{\mu m}]$ & V_DS & EXP & EST \\
\hline $\mathbf{1 0 0}$ & 2 & 1.55 & 1.50 \\
& 5 & 1.91 & 1.80 \\
& 10 & 1.92 & 1.89 \\
\hline $\mathbf{5 0}$ & 2 & 1.20 & 1.30 \\
& 5 & 1.44 & 1.52 \\
& 10 & 1.62 & 1.68 \\
\hline $\mathbf{2 0}$ & 2 & 1.30 & 1.22 \\
& 5 & 1.79 & 1.70 \\
& 10 & 1.64 & 1.57 \\
\hline
\end{tabular}

\subsubsection{Threshold Voltage Determination}

The last performance assessment of the proposed estimation method concerned the determination of the voltage intervals corresponding to the different operating regimes of the OTFT devices. Linear and saturation operating zones were evaluated according to

$$
\begin{gathered}
V_{\mathrm{ds}}<V_{\mathrm{ds}}-V_{\mathrm{t}} \quad \text { linear zone } \\
V_{\mathrm{ds}}>V_{\mathrm{ds}}-V_{\mathrm{t}} \quad \text { saturation zone }
\end{gathered}
$$

The interval amplitude can be achieved by evaluating the threshold voltage $V_{t}$; to this aim, the ratio method was exploited. In particular, the transconductance was first calculated as the ratio between the point-to-point differences of both estimated drain current and gate voltage. Successively, the evolution of the ratio between the drain current and the root square of the transconductance versus the gate voltage is considered. The threshold voltage is finally evaluated as the intersection of the straight-line interpolating the linear zone of the considered curve and the axis of the gate voltage (an example can be seen in Figure 13 for an $\mathrm{N}$-type device). In a similar way, experimental values of the threshold voltage were estimated from the acquired signals of drain current and gate voltage.

The values obtained by means of both proposed method and experimental tests are compared in Table 4 for P-type and N-type devices characterized by a channel length of $100 \mu \mathrm{m}$. Regarding the other comparisons, for the threshold voltage, a remarkable concurrence was experienced.

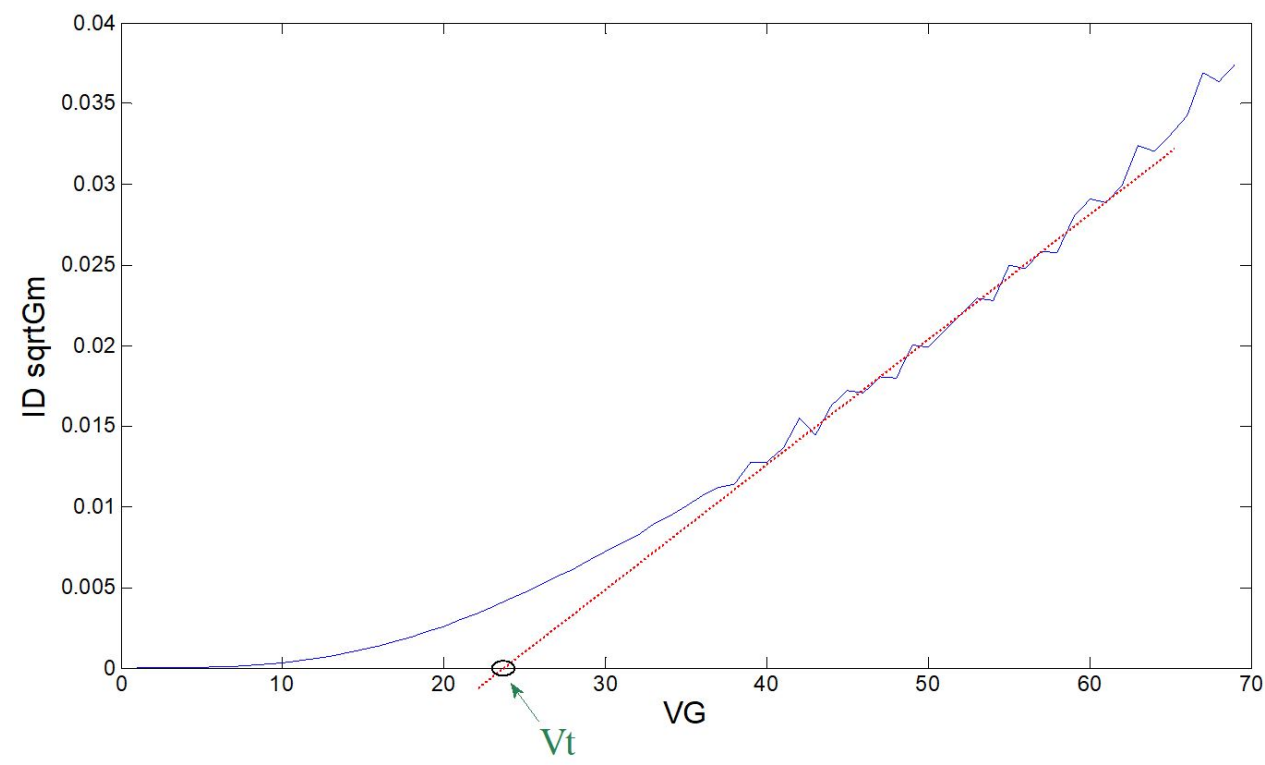

Figure 13. Threshold voltage evaluation. 
Table 4. Comparison between PMOS and NMOS device.

\begin{tabular}{ccccc}
\hline V_DS[V] & \multicolumn{2}{c}{ PMOS [V] } & \multicolumn{2}{c}{ NMOS [V] } \\
\hline $\mathbf{7 0}$ & EXP & EST & EXP & EST \\
\hline $\mathbf{1 0}$ & -22.0 & -22.1 & 22.3 & 22.5 \\
\hline $\mathbf{5}$ & -34.2 & -34.5 & 32.8 & 33.0 \\
\hline $\mathbf{2}$ & -27.7 & -30.0 & 27.8 & 28.9 \\
\hline $\mathbf{1}$ & -18.1 & -18.6 & 25.3 & 26.1 \\
\hline $\mathbf{0 . 5}$ & -13.1 & -13.8 & 25.2 & 25.7 \\
\hline
\end{tabular}

\section{Conclusions}

A method based on MODE approach for the extraction of the parameters characterizing the compact circuit model of OTFT is presented in this paper. In particular, thanks to exploitation of the consecutive application of mono-objective and multi-objective optimization problems involving differential evolution algorithms, it has been possible to estimate model parameters in such a way that they can be used to model transistors characterized by different channel lengths.

The estimation performance of the proposed method has been assessed by means of a number of tests conducted on N-type and P-type OTFTs. The obtained results highlighted that the estimated parameters assured a remarkable concurrence between both experimental and estimated IV characteristics and mobility.

The proposed method was proven to be effective in the extraction of the circuit parameters, thus making their exploitation possible to model the OTFT behavior and to develop useful libraries for hardware design environments.

Author Contributions: Conceptualization, R.S.L.M. and D.R.; methodology, R.S.L.M. and D.R.; software, D.R., F.d.P. and E.C.; validation, L.A., F.d.P. and E.C.; investigation, G.d.A., F.d.P. and E.C.; resources, L.A.; writing—original draft preparation, G.d.A., F.d.P. and E.C.; writing—review and editing, R.S.L.M., G.d.A. and D.R.; supervision, L.A., R.S.L.M. and D.R. All authors have read and agreed to the published version of the manuscript.

Funding: This research received no external funding.

Conflicts of Interest: The authors declare no conflict of interest.

\section{References}

1. Halik, M.; Klauk, H.; Zschieschang, U.; Schmid, G.; Dehm, C.; Schütz, M.; Maisch, S.; Effenberger, F.; Brunnbauer, M.; Stellacci, F. Low-voltage organic transistors with an amorphous molecular gate dielectric. Nature 2004, 431, 963-966. [CrossRef] [PubMed]

2. Ha, Y.G.; Jeong, S.; Wu, J.; Kim, M.G.; Dravid, V.P.; Facchetti, A.; Marks, T.J. Flexible low-voltage organic thin-film transistors enabled by low-temperature, ambient solution-processable inorganic/organic hybrid gate dielectrics. J. Am. Chem. Soc. 2010, 132, 17426-17434. [CrossRef] [PubMed]

3. He, Z.; Chen, J.; Keum, J.K.; Szulczewski, G.; Li, D. Improving performance of TIPS pentacene-based organic thin film transistors with small-molecule additives. Org. Electron. 2014, 15, 150-155. [CrossRef]

4. Ye, R.; Baba, M.; Suzuki, K.; Mori, K. Improved performance of fluorinated copper phthalocyanine thin film transistors using an organic pn junction: Effect of copper phthalocyanine film thickness. Thin Solid Films 2009, 517, 3001-3004. [CrossRef]

5. Lee, C.T.; Lin, Y.M. Performance improvement mechanisms of pentacene-based organic thin-film transistors using TPD buffer layer. Org. Electron. 2013, 14, 1952-1957. [CrossRef]

6. Fukuda, K.; Sekine, T.; Kobayashi, Y.; Kumaki, D.; Itoh, M.; Nagaoka, M.; Toda, T.; Saito, S.; Kurihara, M.; Sakamoto, M.; et al. Stable organic thin-film transistors using full solution-processing and low-temperature sintering silver nanoparticle inks. Org. Electron. 2012, 13, 1660-1664. [CrossRef]

7. Gui, H.; Wei, B.; Wang, J. Inserting a Mn-doped TiO2 layer for improving performance of pentacene organic thin-film transistors. Org. Electron. 2014, 15, 3349-3353. [CrossRef]

8. Bae, J.H.; Choi, Y. Reduction of the trap density at the organic-organic interface and resultant gate-bias dependency of the mobility in an organic thin-film transistor. Solid-State Electron. 2012, 72, 44-47. [CrossRef] 
9. Gold, H.; Haase, A.; Fian, A.; Prietl, C.; Striedinger, B.; Zanella, F.; Marjanović, N.; Ferrini, R.; Ring, J.; Lee, K.D.; et al. Self-aligned flexible organic thin-film transistors with gates patterned by nano-imprint lithography. Org. Electron. 2015, 22, 140-146. [CrossRef]

10. Hoth, C.N.; Schilinsky, P.; Choulis, S.A.; Brabec, C.J. Printing highly efficient organic solar cells. Nano Lett. 2008, 8, 2806-2813. [CrossRef] [PubMed]

11. Pei, K.; Ren, X.; Zhou, Z.; Zhang, Z.; Ji, X.; Chan, P.K.L. A High-Performance Optical Memory Array Based on Inhomogeneity of Organic Semiconductors. Adv. Mater. 2018, 30, 1706647. [CrossRef] [PubMed]

12. Fujisaki, Y.; Sato, H.; Fujikake, H.; Inoue, Y.; Tokito, S.; Kurita, T. Liquid crystal display cells fabricated on plastic substrate driven by low-voltage organic thin-film transistor with improved gate insulator and passivation layer. Jpn. J. Appl. Phys. 2005, 44, 3728. [CrossRef]

13. Dodabalapur, A. Organic and polymer transistors for electronics. Mater. Today 2006, 9, 24-30. [CrossRef]

14. Kraus, R.; Mattausch, H.J. Status and trends of power semiconductor device models for circuit simulation. IEEE Trans. Power Electron. 1998, 13, 452-465. [CrossRef]

15. Ibrahim, D. An overview of soft computing. Procedia Comput. Sci. 2016, 102, 34-38. [CrossRef]

16. Deb, K. Multi-objective optimisation using evolutionary algorithms: An introduction. In Multi-Objective Evolutionary Optimisation for Product Design and Manufacturing; Springer: Berlin/Heidelberg, Germany, 2011; pp. 3-34.

17. Moreno, P.; Picos, R.; Roca, M.; Garcia-Moreno, E.; Iniguez, B.; Estrada, M. Parameter extraction method using genetic algorithms for an improved OTFT compact model. In Proceedings of the IEEE 2007 Spanish Conference on Electron Devices, Madrid, Spain, 31 January-2 February 2007; pp. 64-67.

18. Beyer, H.G. The Theory of Evolution Strategies; Springer Science \& Business Media: New York, NY, USA, 2001.

19. Corne, D.; Dorigo, M.; Glover, F.; Dasgupta, D.; Moscato, P.; Poli, R.; Price, K.V. New Ideas in Optimization; McGraw-Hill Ltd.: London, UK, 1999.

20. Eberhart, R.; Kennedy, J. A new optimizer using particle swarm theory. In Proceedings of the IEEE Sixth International Symposium on Micro Machine and Human Science (MHS'95), Nagoya, Japan, 4-6 October 1995; pp. 39-43.

21. Romero, A.; González, J.; Jiménez-Tejada, J.A. Constrained many-objective evolutionary extraction procedure for an OTFT compact model including contact effects. In Proceedings of the IEEE 2018 Spanish Conference on Electron Devices (CDE), Salamanca, Spain, 14-16 November 2018; pp. 1-4.

22. Babu, B.; Gujarathi, A.M. Multi-objective differential evolution (mode) algorithm for multi-objective optimization: Parametric study on benchmark test problems. J. Future Eng. Technol. 2007, 3, 47-59. [CrossRef]

23. Iwan, M.; Akmeliawati, R.; Faisal, T.; Al-Assadi, H.M. Performance comparison of differential evolution and particle swarm optimization in constrained optimization. Procedia Eng. 2012, 41, 1323-1328. [CrossRef]

24. Vesterstrom, J.; Thomsen, R. A comparative study of differential evolution, particle swarm optimization, and evolutionary algorithms on numerical benchmark problems. In Proceedings of the IEEE 2004 Congress on Evolutionary Computation (IEEE Cat. No. 04TH8753), Portland, OR, USA, 19-23 June 2004; Volume 2, pp. 1980-1987.

25. Jang, J.; Lee, S. A Fundamental Reason for the Need of Two Different Semiconductor Technologies for Complementary Thin-Film Transistor Operations. Crystals 2019, 9, 603. [CrossRef]

26. Han, W.; Yoshida, H.; Ueno, N.; Kera, S. Electron affinity of pentacene thin film studied by radiation-damage free inverse photoemission spectroscopy. Appl. Phys. Lett. 2013,103,171_1. [CrossRef]

27. Dodabalapur, A.; Torsi, L.; Katz, H. Organic transistors: Two-dimensional transport and improved electrical characteristics. Science 1995, 268, 270-271. [CrossRef] [PubMed]

28. Muller, R.S.; Kamins, T.I. Device Electronics For Integrated Circuits, 3rd ed.; John Wiley \& Sons: New York, NY, USA, 2003.

29. Abdinia, S.; van Roermund, A.H.M.; Cantatore, E. OTFT Modelling and Characteristics. In Design of Organic Complementary Circuits and Systems on Foil; Springer International Publishing: Cham, Switzerland, 2015; pp. 27-45._3. [CrossRef] 